\title{
Next-Generation Accurate, Transferable, and Polarizable PHAHST Potentials for Material Simulations
}

\author{
Adam Hogan and Brian Space* \\ Department of Chemistry, University of South Florida, 4202 E. Fowler Ave., CHE205, \\ Tampa, Florida 33620-5250, United States \\ E-mail: bspace@usf.edu
}

\section{S1 Noble Gas Mixing Rule Comparison}

As an initial validation of the proposed potentials and mixing rules, well minimums, $\mathrm{R}_{m}$, and well depths, $\mathrm{D}_{e}$, were computed for the PHAHST models and compared to experiment and the standard Lorentz-Berthelot (LB) mixing rules employed in Lennard-Jones simulations. ${ }^{1}$ Root mean square errors (RMSE) for the PHAHST mixing rules were significantly lower than the standard LB mixing rules. 
Table S1: Well minimums, $\mathrm{R}_{m}$ in $\AA$, and well depths, $\mathrm{D}_{e}$ in $\mathrm{K} / k_{B}$, for the PHAHST potentials and experimental data as well as percent differences, $\%$ Diff, for the PHAHST mixing and the Lorentz-Berthelot (LB) mixing rules. ${ }^{1}$

\begin{tabular}{|l|l|l|l|l|l|l|l|l|}
\hline Pair & $\begin{array}{l}\mathrm{R}_{m} \\
\text { (this) }\end{array}$ & $\begin{array}{l}\mathrm{D}_{e} \\
\text { (this) }\end{array}$ & $\begin{array}{l}\mathrm{R}_{m} \\
\text { (Exp.) }\end{array}$ & $\begin{array}{l}\mathrm{D}_{e} \\
(\text { Exp.) }\end{array}$ & $\begin{array}{l}\% \text { Diff } \\
\mathrm{R}_{m} \\
(\text { this })\end{array}$ & $\begin{array}{l}\% \text { Diff } \\
\mathrm{D}_{e} \\
\text { (this) }\end{array}$ & $\begin{array}{l}\% \text { Diff } \\
\mathrm{R}_{m} \\
(\mathrm{LB})\end{array}$ & $\begin{array}{l}\% \text { Diff } \\
\mathrm{D}_{e} \\
(\mathrm{LB})\end{array}$ \\
\hline $\mathrm{He}-\mathrm{He}$ & 2.96 & 10.07 & 2.93 & 10.44 & $1.04 \%$ & $-3.54 \%$ & - & - \\
\hline $\mathrm{He}-\mathrm{Ne}$ & 3.00 & 20.79 & 3.02 & 19.44 & $-0.68 \%$ & $6.94 \%$ & $-0.3 \%$ & $7.7 \%$ \\
\hline $\mathrm{He}-\mathrm{Ar}$ & 3.40 & 30.47 & 3.46 & 30.01 & $-1.78 \%$ & $1.55 \%$ & $-3.4 \%$ & $28.1 \%$ \\
\hline $\mathrm{He}-\mathrm{Kr}$ & 3.65 & 31.08 & 3.67 & 31.05 & $-0.47 \%$ & $0.08 \%$ & $-5.4 \%$ & $46.4 \%$ \\
\hline $\mathrm{He}-\mathrm{Xe}$ & 3.90 & 30.75 & 3.97 & 29.77 & $-1.66 \%$ & $3.28 \%$ & $-8.1 \%$ & $79.7 \%$ \\
\hline $\mathrm{Ne}-\mathrm{Ne}$ & 3.07 & 41.92 & 3.09 & 42.00 & $-0.80 \%$ & $-0.18 \%$ & - & - \\
\hline $\mathrm{Ne}-\mathrm{Ar}$ & 3.46 & 67.92 & 3.50 & 64.17 & $-1.23 \%$ & $5.84 \%$ & $-2.1 \%$ & $20.2 \%$ \\
\hline $\mathrm{Ne}-\mathrm{Kr}$ & 3.30 & 71.26 & 3.66 & 67.32 & $-1.74 \%$ & $5.85 \%$ & $-3.1 \%$ & $35.4 \%$ \\
\hline $\mathrm{Ne}-\mathrm{Xe}$ & 3.89 & 73.07 & 3.92 & 67.25 & $-0.75 \%$ & $8.65 \%$ & $-4.8 \%$ & $59.5 \%$ \\
\hline $\mathrm{Ar}-\mathrm{Ar}$ & 3.70 & 141.9 & 3.76 & 141.5 & $-1.60 \%$ & $0.30 \%$ & - & - \\
\hline $\mathrm{Ar}-\mathrm{Kr}$ & 3.88 & 166.3 & 3.89 & 165.8 & $-0.21 \%$ & $0.30 \%$ & $-0.1 \%$ & $0.9 \%$ \\
\hline $\mathrm{Ar}-\mathrm{Xe}$ & 4.08 & 185.3 & 4.11 & 182.6 & $-0.69 \%$ & $1.51 \%$ & $-1.2 \%$ & $7.8 \%$ \\
\hline $\mathrm{Kr}-\mathrm{Kr}$ & 4.00 & 201.6 & 4.01 & 197.8 & $-0.21 \%$ & $1.90 \%$ & - & - \\
\hline $\mathrm{Kr}-\mathrm{Xe}$ & 4.19 & 233.1 & 4.21 & 225.4 & $-0.58 \%$ & $3.43 \%$ & $-0.7 \%$ & $3.3 \%$ \\
\hline $\mathrm{Xe}-\mathrm{Xe}$ & 4.36 & 280.6 & 4.36 & 274.0 & $-0.09 \%$ & $2.40 \%$ & - & - \\
\hline $\mathrm{RMSE}$ & & & & & $1.06 \%$ & $4.01 \%$ & $3.11 \%$ & $31.2 \%$ \\
\hline
\end{tabular}




\section{S2 Fragments Used in Fitting Process}

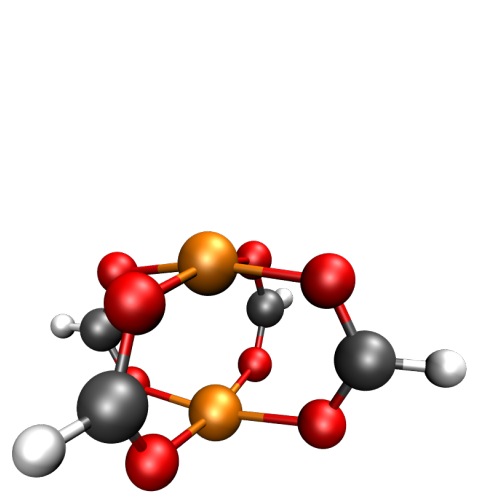

(a) Fragment 1

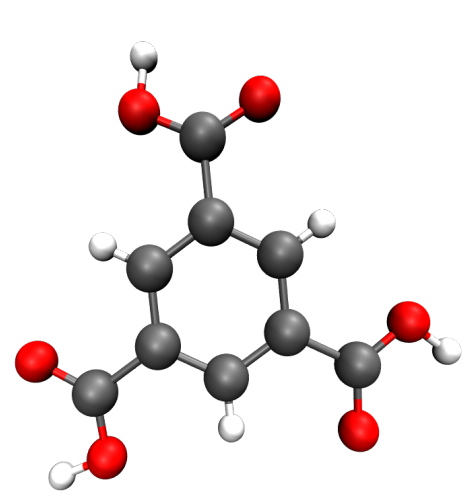

(b) Fragment 2

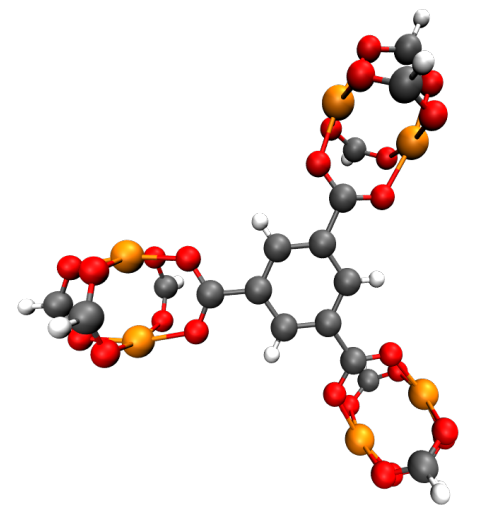

(c) Fragment 3

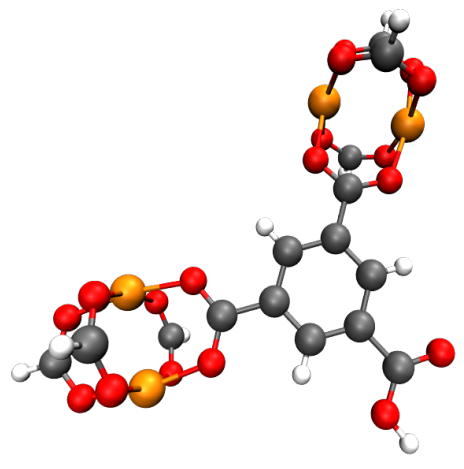

(d) Fragment 4

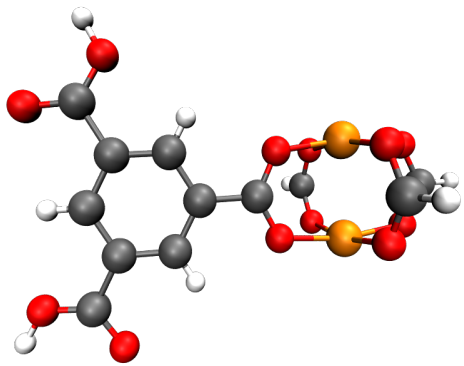

(e) Fragment 5

Figure S1: Gas phase HKUST-1 fragments extracted from the experimental crystal structure used in the material fitting process. 


\section{S3 XDM Parameters}

XDM atomic moment integrals were calculated for fragment 1 using NWChem, the PBE0 functional and the def2-qzvpp basis set. Default a1 and a2 parameters of 0.4186 and 2.6791 were used respectively. Dispersion coefficients were calculated via $\mathrm{C} 6=\frac{1}{2} \alpha\left\langle M_{1}^{2}\right\rangle$, $\mathrm{C} 8=$ $\frac{3}{2} \alpha\left\langle M_{2}^{2}\right\rangle$, and $\mathrm{C} 10=2 \alpha\left\langle M_{3}^{2}\right\rangle+\frac{21}{10} \alpha\left\langle M_{2}^{2}\right\rangle^{2} /\left\langle M_{1}^{2}\right\rangle \cdot{ }^{2,3}$

Table S2: Calculated XDM atomic moment integrals for use in constraining dispersion coefficients to physically reasonable values during the materials fitting process.

\begin{tabular}{|c|c|c|c|}
\hline Atom & $\left\langle M_{1}^{2}\right\rangle$ & $\left\langle M_{2}^{2}\right\rangle$ & $\left\langle M_{3}^{2}\right\rangle$ \\
\hline $\mathrm{Cu}$ & 7.0612840320 & 88.7627561823 & 2362.2083365453 \\
\hline $\mathrm{O}$ & 4.8848635283 & 41.6971454022 & 500.0960034794 \\
\hline $\mathrm{C}$ & 4.9409882783 & 75.8437078276 & 1615.7234406516 \\
\hline $\mathrm{H}$ & 2.2783926941 & 23.4786386467 & 456.2564236433 \\
\hline
\end{tabular}




\section{S4 HKUST-1 Partial Charges}

Partial charges for HKUST-1 were obtained by averaging CHELPG charges of gas phase fragments calculated with NWChem. ${ }^{2}$ The $6-31 \mathrm{G}^{*}$ basis set was used for $\mathrm{C}, \mathrm{H}$, and $\mathrm{O}$ while the LANL2DZ basis set was used for $\mathrm{Cu}$, the relatively poor basis sets produce "overpolarized" charges leading to reasonable agreement with more advanced calculations. ${ }^{4}$

Table S3: Calculated partial charges $\left(\mathrm{e}^{-}\right)$used for HKUST-1 simulations. $\mathrm{C}_{\text {carboxylate }}$ is the carbon atom immediately adjacent to the oxygens, $\mathrm{C}_{\text {aromatic, } 1}$ is the carbon atom immediately adjacent to $\mathrm{C}_{\text {carboxylate, }}$ and $\mathrm{C}_{\text {aromatic, } 2}$ is the carbon atom adjacent to $\mathrm{C}_{\text {aromatic, } 1}$ and the hydrogen atoms.

\begin{tabular}{|c|c|c|}
\hline Atom & Fragment Charge & Periodic Charge \\
\hline $\mathrm{Cu}$ & 1.08076 & 0.93980 \\
\hline $\mathrm{O}$ & -0.72538 & -0.58339 \\
\hline $\mathrm{H}$ & 0.17857 & 0.13953 \\
\hline $\mathrm{C}_{\text {carboxylate }}$ & 0.93474 & 0.72259 \\
\hline $\mathrm{C}_{\text {aromatic }, 1}$ & -0.21244 & -0.09268 \\
\hline $\mathrm{C}_{\text {aromatic }, 2}$ & 0.00951 & -0.07256 \\
\hline
\end{tabular}




\section{References}

(1) Kestin, J.; Knierim, K.; Mason, E. A.; Najafi, B.; Ro, S. T.; Waldman, M. Equilibrium and Transport Properties of the Noble Gases and Their Mixtures at Low Density. J. Phys. Chem. Ref. Data 1984, 13, 229-303.

(2) Valiev, M.; Bylaska, E. J.; Govind, N.; Kowalski, K.; Straatsma, T. P.; Van Dam, H. J.; Wang, D.; Nieplocha, J.; Apra, E.; Windus, T. L. NWChem: A Comprehensive and Scalable Open-Source Solution for Large Scale Molecular Simulations. Comput. Phys. Commun. 2010, 181, 1477-1489.

(3) Becke, A. D.; Johnson, E. R. Exchange-Hole Dipole Moment and the Dispersion Interaction Revisited. J. Chem. Phys. 2007, 127, 154108.

(4) Stern, A. C.; Belof, J. L.; Eddaoudi, M.; Space, B. Understanding Hydrogen Sorption in a Polar Metal-Organic Framework with Constricted Channels. J. Chem. Phys. 2012, $136,034705$. 